\title{
Primary peritoneal carcinosarcoma arising from the Douglas pouch: A case report
}

\author{
TAKAHIRO KOYANAGI ${ }^{1,2}$, YOKO TO $^{1}$, MIHO ANDO $^{1}$, SAKIKO MATSUOKA $^{1}$, SUMIE NAKAMURA ${ }^{1}$, \\ MAKI GOTO $^{1}$, HARUHIKO KONDO ${ }^{1}$, FUYUKI EGUCHI $^{1}$ and HIROSHI TSUJIOKA ${ }^{1}$ \\ ${ }^{1}$ Department of Obstetrics and Gynecology, ASO Iizuka Hospital, Iizuka, Fukuoka 820-8505; ${ }^{2}$ Department of Obstetrics \\ and Gynecology, School of Medicine, Jichi Medical University, Shimotsuke, Tochigi 329-0498, Japan
}

Received June 23, 2018; Accepted August 17, 2018

DOI: $10.3892 / \mathrm{mco} .2018 .1711$

\begin{abstract}
Primary peritoneal carcinosarcoma is extremely rare and only few cases have been reported in the literature to date. We herein present a case of carcinosarcoma of the Douglas pouch in a 73-year-old Japanese woman. The patient complained of fever and lower abdominal pain, and a large pelvic mass ( $>10 \mathrm{~cm}$ in diameter) was detected, with rectal invasion. Laparotomy was performed and revealed a left ovarian abscess and a Douglas pouch mass; however, there was no obvious tumor involvement of the bilateral ovaries or uterus. The patient underwent total abdominal hysterectomy, bilateral salpingo-oophorectomy and tumor debulking, with a reduction rate of $\sim 30 \%$. Sigmoid colostomy was also performed due to the deep and wide rectal invasion. Histologically, the tumor was composed of a mixture of ovarian high-grade serous carcinoma and spindle-cell sarcoma mimicking leiomyosarcoma. Immunohistochemically, the serous carcinoma component was positive for cytokeratin (CK)7, Wilms' tumor-1 and p53 (null type), while CDX-2 and CK20 were negative. The spindle-cell sarcoma component was positive for vimentin and $\alpha$-smooth muscle actin. The present case was diagnosed as carcinosarcoma of the homologous type derived from the peritoneum in the Douglas pouch. The patient received several courses of combination chemotherapy with paclitaxel, carboplatin and bevacizumab, and achieved complete remission. The principal treatment for such cases is surgery, and several chemotherapeutic regimens, including paclitaxel and carboplatin, or cisplatin and ifosfamide, have been reported. The accumulation of more clinical cases is crucial for understanding the clinicopathological characteristics of these rare tumors and establishing effective therapeutic strategies.
\end{abstract}

Correspondence to: Dr Hiroshi Tsujioka, Department of Obstetrics and Gynecology, ASO Iizuka Hospital, 3-83 Yoshio-machi, Iizuka, Fukuoka 820-8505, Japan

E-mail: htsujiokah1@aih-net.com

Key words: bevacizumab, carcinosarcoma, chemotherapy, Douglas pouch, surgery

\section{Introduction}

Carcinosarcoma, also referred to as malignant mixed Müllerian tumor (MMMT), which contains both carcinomatous and sarcomatous elements, generally arises in the female genital tract. Extragenital carcinosarcoma is rare and several cases of carcinosarcoma occurring in the retroperitoneum (1), mesentery $(2,3)$ and lesser omentum (4) have been reported to date. However, carcinosarcoma arising from the Douglas pouch is extremely rare, with only 2 such cases reported in the English literature $(5,6)$.

The aim of the present study was to present a case of carcinosarcoma arising from the Douglas pouch and discuss the clinicopathological characteristics and therapeutic management of this rare tumor.

\section{Case report}

A 73-year-old woman (gravida 6, para 4) presented with fever and lower abdominal pain. The patient's medical history included hypertension and hyperlipidemia. A vaginal examination revealed purulent discharge and a fist-sized soft tumor was palpable in the pelvis. Pelvic ultrasound examination revealed an irregular round mosaic mass $>10 \mathrm{~cm}$ in greatest diameter. Magnetic resonance imaging examination of the pelvis revealed a large pelvic mass with abscess formation invading the rectum, with enlarged bilateral iliac lymph nodes. The serum carbohydrate antigen 125 level was $334 \mathrm{U} / \mathrm{ml}$. A computed tomography scan confirmed enlargement of Virchow's lymph node. Since left ovarian cancer was suspected, laparotomy was performed. Although a left ovarian abscess and a Douglas pouch mass were detected in the abdominal cavity, there was no obvious tumor involvement of the bilateral ovaries or uterus. Peritoneal dissemination, including the omentum, was not observed. The patient underwent total abdominal hysterectomy, bilateral salpingo-oophorectomy and tumor debulking, with a reduction rate of $\sim 30 \%$, as the tumor firmly adhered to the pelvic wall, uterus and rectum. Sigmoid colostomy was also performed to prevent obstructive ileus due to the deep and wide rectal invasion. The patient received several courses of combination chemotherapy with paclitaxel, carboplatin and bevacizumab, according to the standard adjuvant chemotherapy guidelines for ovarian cancer (7). 
Each drug was administered triweekly: Paclitaxel, $175 \mathrm{mg} / \mathrm{m}^{2}$; carboplatin, AUC 6 and bevacizumab, $15 \mathrm{mg} / \mathrm{kg}$. After three courses, complete remission was achieved and no recurrence has been detected during follow-up to date (data not shown).

Histologically, the pelvic tumor was composed of a mixture of serous carcinoma and spindle-cell sarcoma with necrosis and hemorrhage. The epithelial component displayed tubular, papillary, or cribriform proliferation of severely atypical cells with mitotic figures, mimicking ovarian high-grade serous carcinoma. The adjacent stromal component consisted of atypical spindled cells with severe nuclear atypia and mitotic figures. The spindle-cell sarcoma element partly exhibited myxomatous changes. Although a left ovarian abscess was identified, tumor cells were not detected in the bilateral ovaries or uterus.

Immunohistochemically, the serous carcinoma component was positive for cytokeratin (CK)7, Wilms' tumor-1 and p53 (the null type), while CDX-2 and CK20 were negative. The spindle-cell sarcoma component was positive for vimentin and $\alpha$-smooth muscle actin. p53 was also positive (the null type) in the sarcoma component. The case was diagnosed as carcinosarcoma of the homologous type primarily derived from the peritoneum in the Douglas pouch.

\section{Discussion}

A rare case of primary peritoneal carcinosarcoma of the homologous type arising in the Douglas pouch was encountered and successfully treated with adjuvant chemotherapy, including a molecular-targeting agent.

Carcinosarcoma of the female genital tract, also referred to as MMMT, is divided into two groups, homologous and heterologous types, according to the histological characteristics of the sarcomatous element. The malignant mesenchymal component of the tumor is described as homologous or heterologous (8). The homologous type consists of tissues indigenous to Müllerian structures (e.g., resembling endometrial stromal sarcoma, fibrosarcoma, or leiomyosarcoma). If the sarcomatous component contains elements not normally found in the Müllerian structures (e.g., cartilaginous, osseous, or rhabdomyocytic), it is described as heterologous. The epithelial and mesenchymal elements in these tumors are generally randomly admixed. Both are high-grade, while the mesenchymal component may occasionally be composed of relatively bland spindle cells. In the present case, the epithelial component resembled ovarian high-grade serous carcinoma and the mesenchymal element mimicked leiomyosarcoma. Thus, the patient was diagnosed with carcinosarcoma of the homologous type.

Carcinosarcoma is an aggressive tumor and it may be resistant to conventional chemotherapy. Since primary peritoneal carcinosarcoma is rare, there are no established treatment strategies or guidelines and treatment decisions are based on each individual diagnosis. In the present case, combination chemotherapy with paclitaxel and carboplatin was initiated, which is commonly used for epithelial ovarian cancer. The molecular-targeting agent bevacizumab, a monoclonal antibody against vascular endothelial growth factor (VEGF), was also included in the treatment regimen. This regimen was proven to be markedly effective and a complete

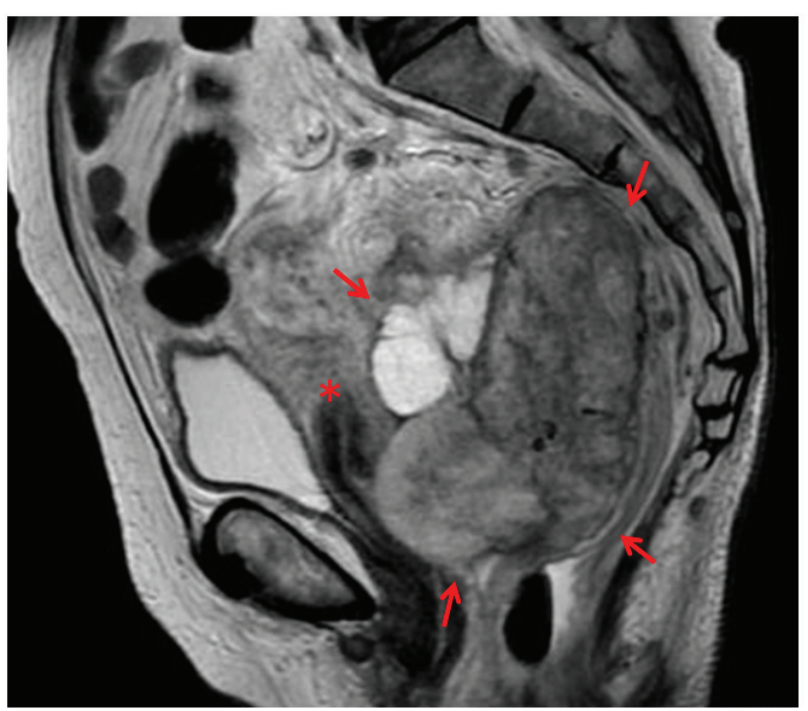

Figure 1. T2-weighed magnetic resonance imaging of the pelvis revealed a large pelvic solid tumor. Arrows indicate the tumor, whereas the high-intensity area appears to correspond to abscess formation. The uterus was compressed by the pelvic tumor (asterisk).

response was achieved after three courses. The patient has remained disease-free by continuing this regimen. To the best of our knowledge, this appears to be the first medical case report of Douglas pouch carcinosarcoma successfully treated with chemotherapy including anti-VEGF therapy with bevacizumab.

Some reviews have been published on the primary treatment and prognostic factors of Müllerian carcinosarcomas $(9,10)$. Based on these reviews, platinum-based chemotherapy is the mainstay of adjuvant systemic treatment, and the addition of paclitaxel or ifosfamide to platinum is recommended as first-line treatment. However, these cases include several carcinosarcoma cases of the ovaries and fallopian tubes. Thus, selective analyses of primary peritoneal carcinosarcoma cases are needed in future studies.

Extragenital carcinosarcomas mostly occur in the pelvic peritoneum (11), whereas they rarely develop in the retroperitoneum (1), mesentery $(2,3)$ and lesser omentum (4). Carcinosarcoma arising from the Douglas pouch is extremely rare, this being the third case reported in the English literature to date $(5,6)$. Due to its rarity, there are difficulties with making an accurate preoperative diagnosis of primary peritoneal carcinosarcoma. In the present case, the preoperative diagnosis was left ovarian cancer and the tumor was found to be located in the Douglas pouch after laparotomy. Clinicians must be aware of extragenital malignancies when a large pelvic mass is detected clinically.

The etiology and tumorigenesis of primary peritoneal carcinosarcoma remain unknown. The origin of the present tumor was hypothesized to be peritoneal multipotent cells or misplaced Müllerian duct remnants in the Douglas pouch (12). However, further studies are required to elucidate its cellular origin.

The principal treatment for primary peritoneal carcinosarcoma is optimal surgery and subsequent systemic chemotherapy. Despite its rarity, the accumulation of more 

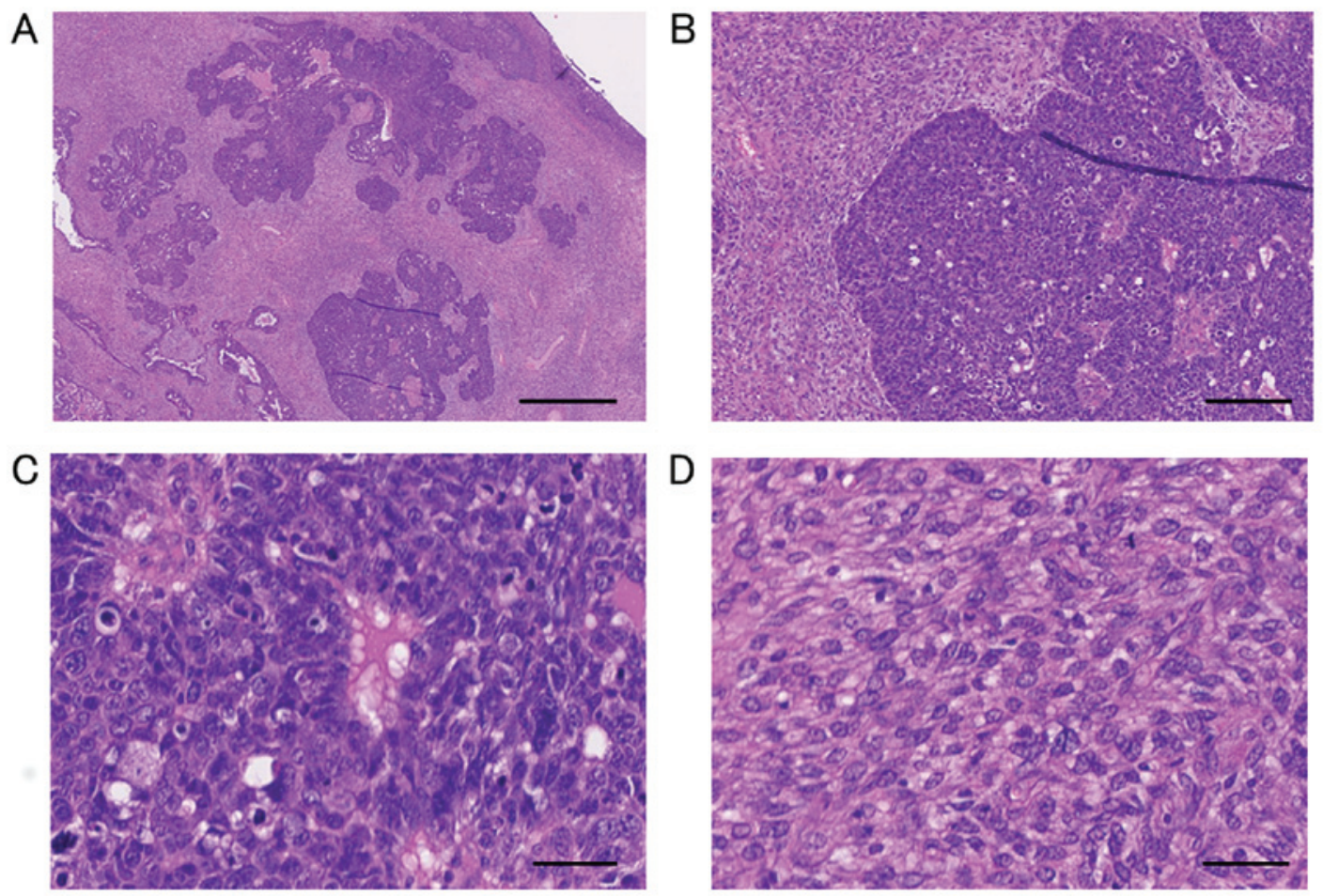

Figure 2. (A and B) On examination of hematoxylin and eosin-stained sections, the pelvic tumor consisted of atypical epithelial elements and atypical spindle cell components. (C) High-power view of the epithelial component. The atypical epithelial component displayed solid and tubular proliferation of cells with severe nuclear atypia and mitotic figures, mimicking ovarian high-grade serous carcinoma. (D) High-power view of the mesenchymal component. The stromal component consisted of atypical spindle cells with severe nuclear atypia and mitotic figures, mimicking leiomyosarcoma. Myxomatous changes were also partly observed. Scale bar, (A) $1 \mathrm{~mm}$, (B) $200 \mu \mathrm{m}$ and (C and D) $50 \mu \mathrm{m}$.
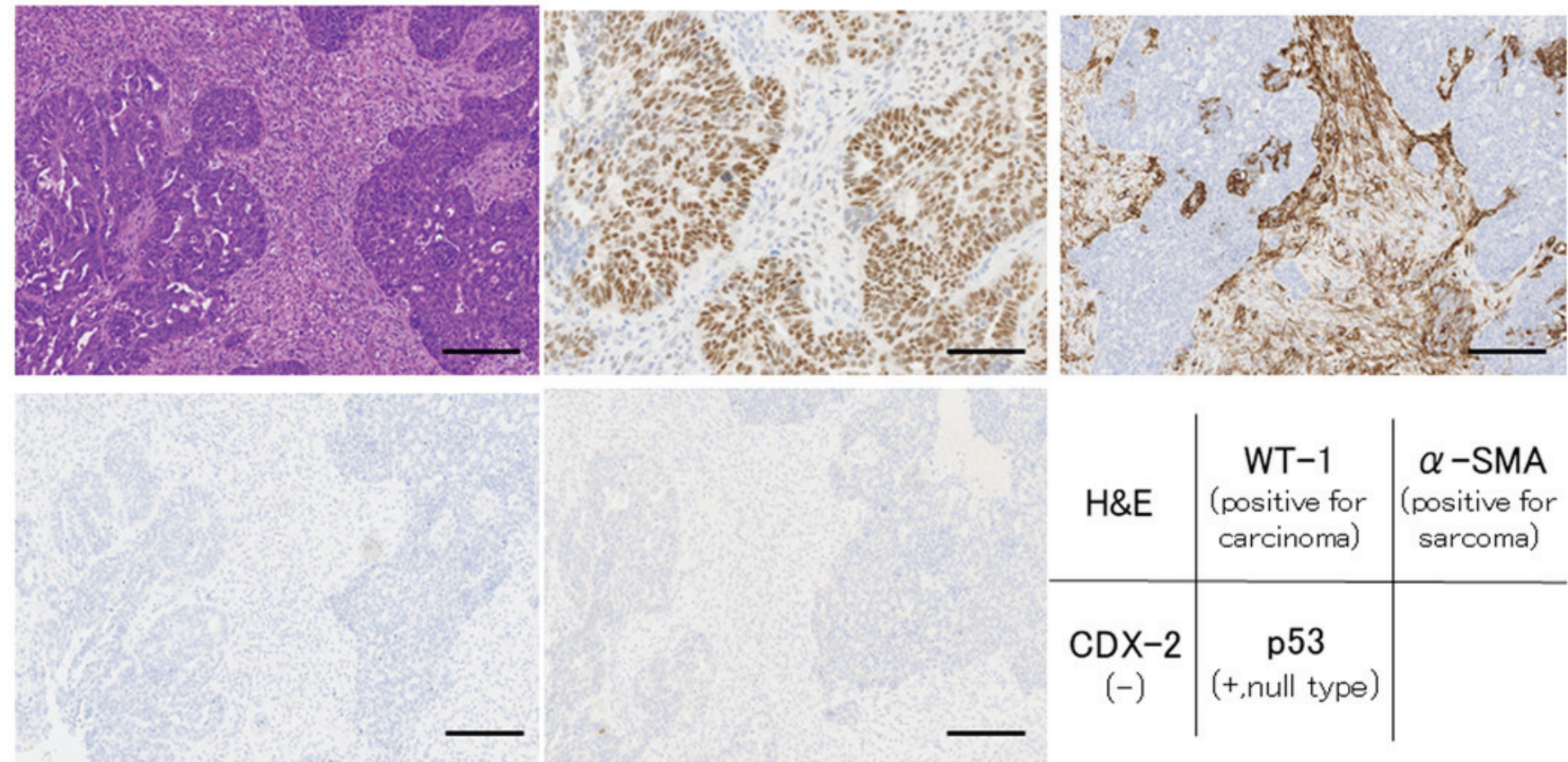

Figure 3. Immunohistochemical analyses of the pelvic tumor. As shown in the lower right panel, the epithelial component was positive for Wilms' tumor (WT)-1 and negative for CDX-2. The mesenchymal element was positive for $\alpha$-smooth muscle actin (SMA). p53 was positive (null type) in both components. Scale bar, $200 \mu \mathrm{m}$ (except for WT-1: $100 \mu \mathrm{m})$. H\&E, hematoxylin and eosin staining.

clinical cases is crucial for elucidating the clinicopathological characteristics of this rare tumor and establishing effective therapeutic strategies.

\section{Acknowledgements}

Not applicable. 


\section{Funding}

No funding was received.

\section{Authors' contributions}

TK, YT and HT diagnosed, investigated and managed the patient. TK, YT, HK and HT determined the medical significance of this case and wrote the manuscript. MA, SM, SN, MG and FE provided advice in managing the patient's treatment and preparing the manuscript. All the authors have read and approved the final version of this manuscript.

\section{Availability of data and materials}

Not applicable.

\section{Ethics approval and consent to participate}

Not applicable.

\section{Patient consent for publication}

The authors obtained written informed consent for publication of the case details, and patient anonymity has been preserved.

\section{Competing interests}

The authors declare that they have no competing interests to disclose.

\section{References}

1. Shintaku M and Matsumoto T: Primary mullerian carcinosarcoma of the retroperitoneum: report of a case. Int J Gynecol Pathol 29: 191-195, 2001.
2. Mikami M, Kuwabara Y, Tanaka K, Komiyama S, Ishikawa M and Hirose T: Malignant mixed müllerian tumor of primary mesenteric origin. Int J Gynecol Cancer 15: 1249-1253, 2005.

3. Cokelaere K, Michielsen P, De Vos R and Sciot R: Primary mesenteric malignant mixed mesodermal (müllerian) tumor with neuroendocrine differentiation. Mod Pathol 14: 515-520, 2001.

4. Wang B, Ren KW, Yang YC, Wan DL, Li XJ, Zhai ZL, Zhang LL and Zheng SS: Carcinosarcoma of the Lesser Omentum: A Unique Case Report and Literature Review. Medicine (Baltimore) 95: e3246, 2016.

5. Terada T: Carcinosarcoma in the pouch of Douglas. Arch Gynecol Obstet 281: 345-348, 2010.

6. Kataoka TR, Shinoda H, Yanagawa M, Kamiura S, Nishizawa Y and Hongyo T: Heterologous carcinosarcoma of Douglas' pouch with adenocarcinomas of the fallopian tube and the peritoneal cavity. Pathology 40: 641-645, 2008.

7. Aravantinos G, Pectasides D :Bevacizumab in combination with chemotherapy for the treatment of advanced ovarian cancer: a systematic review. J Ovarian Res 7: 57, 2014.

8. Sumathi VP, Murnaghan M, Dobbs SP and McCluggage WG Extragenital müllerian carcinosarcoma arising from the peritoneum: report of two cases. Int J Gynecol Cancer 12: 764-767, 2002.

9. Lu CH, Chen IH, Chen YJ, Wang KL, Qiu JT, Lin H, Lin WC, Liou WS, Huang YF, Lin YS, Tee YT and Hung YC: Primary treatment and prognostic factors of carcinosarcoma of the ovary, fallopian tube, and peritoneum: a Taiwanese Gynecologic Oncology Group Study. Int J Gynecol Cancer 24: 506-512, 2014

10. Rauh-Hain JA, Birrer M and Del Carmen MG: 'Carcinosarcoma of the ovary, fallopian tube, and peritoneum: Prognostic factors and treatment modalities'. Gynecol Oncol 142: 248-254, 2016.

11. Shen DH, Khoo US, Xue WC, Ngan HY, Wang JL, Liu VW, Chan YK and Cheung AN: Primary peritoneal malignant mixed Müllerian tumors. A clinicopathologic, immunohistochemical, and genetic study. Cancer 91: 1052-1060, 2001.

12. Mira JL, Fenoglio-Preiser CM and Husseinzadeh N: Malignant mixed müllerian tumor of the extraovarian secondary müllerian system. Report of two cases and review of the English literature. Arch Pathol Lab Med 119: 1044-1049, 1995. 